BULLETIN (New Series) OF THE

AMERICAN MATHEMATICAL SOCIETY

Volume 39, Number 4, Pages 533-555

S 0273-0979(02)00950-3

Article electronically published on July 8, 2002

\title{
FROM DEEP HOLES TO FREE PLANES
}

\author{
CHUANMING ZONG \\ Dedicated to Eli Goodman and Ricky Pollack
}

\begin{abstract}
During the last decades, by applying techniques from Number Theory, Combinatorics and Measure Theory, remarkable progress has been made in the study of deep holes, free planes and related topics in packings of convex bodies, especially in lattice ball packings. Meanwhile, some fascinating new problems have been proposed. To stimulate further research in related areas, we will review the main results, some key techniques and some fundamental problems about deep holes, free cylinders and free planes in this paper.
\end{abstract}

\section{BACKGROUND AND EXAMPLES}

Question 0.1. In an equally populated region the government is going to open a large (fixed) number of schools. Of course, a pupil will be happy if he lives in the vicinity of a school. However, no matter how the schools are located, some of the pupils (in the deep holes) are farther away than the others. In this situation, how should the locations of the schools be chosen so as to minimize the schooling distance of those pupils?

Let $E^{n}$ denote the $n$-dimensional Euclidean space and let $\left\|\mathbf{x}_{1}, \mathbf{x}_{2}\right\|$ denote the Euclidean distance between two points $\mathbf{x}_{1}$ and $\mathbf{x}_{2}$. For convenience, we call the minimum distance between distinct points of a set its separation. Based on Question 0.1 it is natural to ask the following question.

Question 0.2. For which discrete planar set $X$ of separation 2 does the function

$$
\rho(X)=\sup _{\mathbf{y} \in E^{2}} \min _{\mathbf{x} \in X}\|\mathbf{y}, \mathbf{x}\|
$$

attain its minimum? What is the minimum?

By a routine argument one can show that the minimum is $2 / \sqrt{3}$ and every optimal set is isometric to

$$
\Lambda_{2}=\left\{z_{1}(2,0)+z_{2}(1, \sqrt{3}): z_{i} \in Z\right\},
$$

where $Z$ indicates the integer ring. In this case, one can triangulate $E^{2}$ into congruent regular triangles of edge length 2 and with all their vertices belonging to $\Lambda_{2}$.

Received by the editors May 31, 2001, and, in revised form, January 1, 2002.

2000 Mathematics Subject Classification. Primary 05B40, 11H31, 52C15, 52C17.

This work is supported by the National Science Foundation of China and a special grant from Peking University. 
Clearly, for a region of a particular shape, some subset of $\Lambda_{2}$ will provide a solution for Question 0.1.

Let $D$ denote the disk $\left\{(x, y): x^{2}+y^{2} \leq 1\right\}$. We will call $D+X$ a packing of $D$ in $E^{2}$ if there is no overlap among all disks $D+\mathbf{x}, \mathbf{x} \in X$. Then one can state Question 0.2 in a more intuitive version.

Question 0.3. For each packing $D+X$ there is a maximum number $r(X)$ such that a circular disk of radius $r(X)$ can be inscribed into $E^{2} \backslash\{D+X\}$. For which set $X$ does the function $r(X)$ attain its minimum? What is the minimum?

It is easy to see that the optimal sets for Question 0.3 are identical with those for Question 0.2 and the minimum in Question 0.3 is $2 / \sqrt{3}-1=0.154700538 \cdots$.

These examples show an intuitive picture for the deep hole problem in $E^{2}$. "Free plane" is a higher-dimensional phenomenon, and therefore it is not easy to provide an intuitive example. Nevertheless, deep holes and free planes are significant concepts in Geometry. First of all, they provide insight which helps us understand the structure of an $n$-dimensional metric space. Second, many important problems (both mathematical and non-mathematical) can be formulated in terms of deep holes and free planes. Third, they are becoming an important and active research area in Packing and Covering.

\section{Notation AND DEFInitions}

Let $C$ denote an $n$-dimensional centrally symmetric convex body centered at the origin o. As usual, its interior, surface and volume are denoted by $\operatorname{int}(C), \partial(C)$ and $v(C)$, respectively. Especially, let $B^{n}$ denote the $n$-dimensional unit ball.

Let $C+\mathbf{x}$ denote the translate of $C$ centered at $\mathbf{x}$, let $C+X$ denote the union of the translates of $C$ centered at the points of $X$ and, for a positive number $\lambda$ and a set $A$ of points, let $\lambda A$ denote the homothetic copy of $A$ with factor $\lambda$. We say $C+X$ is a packing of $C$ in $E^{n}$ if

$$
\left(\operatorname{int}(C)+\mathbf{x}_{i}\right) \cap\left(\operatorname{int}(C)+\mathbf{x}_{j}\right)=\emptyset
$$

holds for any distinct points $\mathbf{x}_{i}$ and $\mathbf{x}_{j}$ of $X$. Then we call $X$ a packing set of $C$. Especially, we call it a packing lattice of $C$ if it is a lattice.

Let $r(C, X)$ be the maximum number (to avoid triviality, we assume $r(C, X)$ is finite) such that

$$
r(C, X) C+\mathbf{p} \subseteq E^{n} \backslash\{\operatorname{int}(C)+X\}
$$

holds for some point $\mathbf{p}$. Such a point is called a deep hole in the packing, and the number $1+r(C, X)$ is called the depth of the deep hole. In other words, a deep hole in a packing $C+X$ is a point where one can add a largest homothetic copy of $C$ to the packing. It follows by symmetry that $(1+r(C, X)) C+X$ is a covering of $E^{n}$; that is

$$
(1+r(C, X)) C+X=E^{n} .
$$

Clearly $r(C, X)$ provides a measure of the efficiency of the packing, and the corresponding deep hole provides a solution for some optimization problem.

Next, define

$$
\gamma(C)=1+\min _{X} r(C, X)
$$


where the minimum is over all packing sets $X$ of $C$, and

$$
\gamma^{*}(C)=1+\min _{\Lambda} r(C, \Lambda)
$$

where the minimum is over all packing lattices $\Lambda$ of $C$. Usually, we call $\gamma(C)$ the simultaneous packing and covering constant of $C$ and call $\gamma^{*}(C)$ the simultaneous lattice packing and lattice covering constant of $C$.

There are many fascinating problems about deep holes. Here we list two as their representatives.

Problem 1.1. Is there an $n$-dimensional centrally symmetric convex body $C$ such that $\gamma^{*}(C) \geq 2$ ?

This problem has a more intuitive formulation which is well-known in Packing and Covering: Is there an n-dimensional convex body $K$ such that one can add a translate of $K$ into every lattice packing $K+\Lambda$ ? For such a convex body, if it exists, the density of its densest lattice packing is strictly less than the density of its densest translative packing. The existence of such convex bodies has been conjectured by many authors. However, no example has been discovered.

Before stating Problem 1.2, let us define $\gamma(C)$ and $\gamma^{*}(C)$ in terms of the Minkowski metric. For a given $C$ we can introduce the Minkowski metric

$$
\|\mathbf{x}, \mathbf{y}\|_{C}=\min \{\lambda: \mathbf{x}-\mathbf{y} \in \lambda C ; \lambda \geq 0\}
$$

into the $n$-dimensional linear space $R^{n}$ (where $R$ indicates the real number field), producing a Minkowski space $M^{n}$. Clearly, $M^{n}$ is nothing else but the Euclidean space when $C=B^{n}$. As usual, for a subset $X$ of $M^{n}$ we call

$$
\inf \left\{\|\mathbf{x}, \mathbf{y}\|_{C}: \mathbf{x} \neq \mathbf{y}, \mathbf{x}, \mathbf{y} \in X\right\}
$$

the separation of $X$. Thus,

$$
1+r(C, X)=\sup _{\mathbf{y} \in M^{n}} \min _{\mathbf{x} \in X}\|\mathbf{y}, \mathbf{x}\|_{C}
$$

holds for every discrete set $X$ of separation 2 in $M^{n}$. Consequently, we have

$$
\gamma(C)=\min _{X} \sup _{\mathbf{y} \in M^{n}} \min _{\mathbf{x} \in X}\|\mathbf{y}, \mathbf{x}\|_{C},
$$

where the outer minimum is over all discrete sets of separation 2 in $M^{n}$, and

$$
\gamma^{*}(C)=\min _{\Lambda} \sup _{\mathbf{y} \in M^{n}} \min _{\mathbf{x} \in \Lambda}\|\mathbf{y}, \mathbf{x}\|_{C},
$$

where the outer minimum is over all packing lattices of $C$.

Problem 1.2. Determine the values of $\gamma(C)$ and $\gamma^{*}(C)$ and their corresponding optimal packing sets for some particular $C$, for example $B^{n}$.

This problem is very important indeed. On the one hand, it creates an active research area in Packing and Covering. On the other hand, it provides a model for many optimization problems. Designing efficient error-correcting codes is such a problem (over a finite field).

A plane $F$ is called a free plane in a packing $C+X$ if it has no intersection with $\operatorname{int}(C)+X$; that is

$$
F \cap(\operatorname{int}(C)+X)=\emptyset .
$$

The existence of free planes is relatively difficult to imagine. However, they do exist, at least in every lattice ball packing. Let $\kappa(C)$ denote the maximal integer $\kappa$ 
such that in every packing $C+X$ there is a $\kappa$-dimensional free plane, and let $\kappa^{*}(C)$ denote the corresponding number when $X$ is limited within lattices. It is obvious that

$$
\kappa(C) \leq \kappa^{*}(C) .
$$

In fact, as we will see from Corollary 6.1,

$$
\kappa(C)=0
$$

holds for every convex body $C$. However, $\kappa^{*}(C)$ is much more complicated and challenging compared with $\kappa(C)$, especially in the ball case. We have the following key problem about free planes.

Problem 1.3. Determine the value of $\kappa^{*}(C)$ for some particular $C$, for example $B^{n}$, and determine the asymptotic order of $\kappa^{*}\left(B^{n}\right)$ for sufficiently large $n$.

Deep holes and free planes are two basic concepts needed to understand packings and coverings of convex bodies. In fact, they describe the key geometric structure of $E^{n} \backslash\{\operatorname{int}(C)+X\}$. At first glance they are rather independent of each other. However, they are closely related by means of a sequence of numbers called covering minima. Determining the values of $\gamma(C), \gamma^{*}(C)$ and $\kappa^{*}(C)$ for a particular centrally symmetric convex body - similar to determining its packing density, covering density and kissing number - is a fascinating but very challenging problem. For more related definitions we refer to [8], 16], [41] or [51].

\section{DeEP holes IN THREE-DIMENSIONAL BALL PACKINGS}

In 1976 L. Fejes Tóth [15] proposed a problem to determine $\gamma\left(B^{n}\right)$ and its corresponding optimal packing sets. In the planar case, it is easy to show that

$$
\gamma\left(B^{2}\right)=\gamma^{*}\left(B^{2}\right)=2 / \sqrt{3}
$$

and every optimal packing set is isometric to $\Lambda_{2}$ (see Section 0). However, as one can predict, the problem turns out to be very hard in higher dimensions.

Let $X=\left\{\mathbf{x}_{1}, \mathbf{x}_{2}, \cdots\right\}$ be a discrete set of points such that $B^{n}+X$ is a packing in $E^{n}$. For every point $\mathbf{x}_{i} \in X$ we define

$$
V\left(\mathbf{x}_{i}\right)=\left\{\mathbf{x}:\left\|\mathbf{x}, \mathbf{x}_{i}\right\| \leq\left\|\mathbf{x}, \mathbf{x}_{j}\right\| ; \mathbf{x}_{j} \in X\right\} .
$$

In other words, $V\left(\mathbf{x}_{i}\right)$ consists of the points which are closer to $\mathbf{x}_{i}$ than to any other point of $X$. Usually $V\left(\mathbf{x}_{i}\right)$ is called the Dirichlet-Voronoi cell associated with $\mathbf{x}_{i}$. It is easy to see that all Dirichlet-Voronoi cells are convex polytopes and

$$
E^{n}=\bigcup V\left(\mathbf{x}_{i}\right) .
$$

In addition, since $B^{n}+X$ is a packing in $E^{n}$, we have

$$
B^{n}+\mathbf{x}_{i} \subset V\left(\mathbf{x}_{i}\right) .
$$

Let $\mathbf{v}$ be a common vertex of two Dirichlet-Voronoi cells $V\left(\mathbf{x}_{i}\right)$ and $V\left(\mathbf{x}_{j}\right)$. By their definitions one can deduce

$$
\left\|\mathbf{v}, \mathbf{x}_{i}\right\|=\left\|\mathbf{v}, \mathbf{x}_{j}\right\|
$$


Therefore we have

$$
1+r\left(B^{n}, X\right)=\sup _{\mathbf{x}_{i} \in X} \sup _{\mathbf{x} \in V\left(\mathbf{x}_{i}\right)}\left\|\mathbf{x}, \mathbf{x}_{i}\right\|
$$

This fact localizes the deep hole problem for a fixed ball packing to its DirichletVoronoi cells. When $X$ is a lattice, all the Dirichlet-Voronoi cells are congruent to each other. Therefore one can determine $r\left(B^{n}, X\right)$ by considering only one cell. In this case, we have

$$
1+r\left(B^{n}, X\right)=\max \|\mathbf{o}, \mathbf{v}\|
$$

where the maximum is over all the vertices $\mathbf{v}$ of $V(\mathbf{o})$.

Let $A_{3}$ and $A_{3}^{*}$ denote the face-centered cubic lattice and the body-centered cubic lattice, respectively. That is

$$
A_{3}=\left\{\sum_{i=1}^{3} z_{i} \mathbf{a}_{i}: z_{i} \in Z\right\},
$$

where $\mathbf{a}_{1}=(1,1,0), \mathbf{a}_{2}=(1,-1,0)$ and $\mathbf{a}_{3}=(0,1,-1) ;$ and

$$
A_{3}^{*}=\left\{\sum_{i=1}^{3} z_{i} \mathbf{a}_{i}^{*}: z_{i} \in Z\right\},
$$

where $\mathbf{a}_{1}^{*}=(2,0,0), \mathbf{a}_{2}^{*}=(0,2,0)$ and $\mathbf{a}_{3}^{*}=(1,1,1)$. By routine computations based on (2.1) it follows that $B^{3}+\sqrt{2} A_{3}$ is a lattice packing in which the depth of the deep holes is $\sqrt{2}$ and $B^{3}+\sqrt{4 / 3} A_{3}^{*}$ is a lattice packing in which the depth of the deep holes is $\sqrt{5 / 3}$.

To solve L. Fejes Tóth's problem in three dimensions, Böröczky 3 in 1986 proved the following theorem. In fact, so far this problem is completely solved only in $E^{2}$ and $E^{3}$.

\section{Theorem 2.1.}

$$
\gamma\left(B^{3}\right)=\gamma^{*}\left(B^{3}\right)=\sqrt{5 / 3}
$$

and every optimal packing set is isometric to $\sqrt{4 / 3} A_{3}^{*}$.

In other words, intuitively speaking, in every ball packing $B^{3}+X$ one can add some small balls of radii at least $\sqrt{5 / 3}-1=0.290994448 \cdots$.

Böröczky's proof is elementary in character, but very complicated. Let $X=$ $\left\{\mathbf{x}_{1}, \mathbf{x}_{2}, \cdots\right\}$ be a discrete set in $E^{3}$ such that $B^{3}+X$ is a packing; let $\mathbf{v}$ be a common vertex of four Dirichlet-Voronoi cells $V\left(\mathbf{x}_{j_{1}}\right), V\left(\mathbf{x}_{j_{2}}\right), V\left(\mathbf{x}_{j_{3}}\right)$ and $V\left(\mathbf{x}_{j_{4}}\right)$; and let $T$ denote the simplex (tetrahedron) with vertices $\mathbf{x}_{j_{1}}, \mathbf{x}_{j_{2}}, \mathbf{x}_{j_{3}}$ and $\mathbf{x}_{j_{4}}$. Usually, such a simplex is called a Delone simplex. It is clear that

$$
\left\|\mathbf{x}_{i}, \mathbf{x}_{j}\right\| \geq 2
$$

whenever $i \neq j$, and for all $k=1,2,3$ and 4 ,

$$
\left\|\mathbf{v}, \mathbf{x}_{j_{k}}\right\|=r
$$

holds for some positive number $r$. 
It can be proved that $r \geq \sqrt{5 / 3}$ if one of the four dihedral angles of $T$ is at most $\pi / 3$. On the other hand, if a face of $V\left(\mathbf{x}_{i}\right)$ has $k$ vertices, then there will be $k$ Delone simplices with a common edge $\mathbf{x}_{i} \mathbf{x}_{j}$ which is perpendicular with the face and the sum of the $k$ corresponding dihedral angles is $2 \pi$. If $1+r\left(B^{3}, X\right)<\sqrt{5 / 3}$, then $k \leq 5$ holds for every face of $V\left(\mathbf{x}_{i}\right)$. Based on these facts, by Euler's formula about the numbers of vertices, edges and faces of a polytope, Theorem 2.1 is proved by considering several cases with respect to the number of faces of $V\left(\mathbf{x}_{i}\right)$.

Remark 1. In $E^{2}$ the optimal sets, irrespective of the circle packing density, the depth of the deep hole in a circle packing or the circle covering density, are identical with $\Lambda_{2}$ up to isometry and homothety. In $E^{3}$, however, the situation is very different. Besides Theorem 2.1, it is well-known that every optimal packing lattice of $B^{3}$ with respect to the packing density is isometric to $\sqrt{2} A_{3}$ (see [8] or [51]) and every optimal covering lattice of $B^{3}$ with respect to the covering density is isometric to $\sqrt{4 / 5} A_{3}^{*}$ (see Bambah [1]).

\section{DeEp holes in LatTice Ball PACKingS}

As usual, we write

$$
\begin{aligned}
& A_{n}=\left\{\left(z_{0}, z_{1}, \cdots, z_{n}\right): z_{i} \in Z ; \sum z_{i}=0\right\}, \\
& D_{n}=\left\{\left(z_{1}, z_{2}, \cdots, z_{n}\right): z_{i} \in Z ; \sum z_{i} \in 2 Z\right\}, \\
& E_{8}=\left\{\left(u_{1}, u_{2}, \cdots, u_{8}\right): 2 u_{i} \in Z ; u_{i}-u_{j} \in Z ; \sum u_{i} \in 2 Z\right\}, \\
& E_{7}=\left\{\mathbf{u}: \mathbf{u} \in E_{8} ; \sum u_{i}=0\right\} \\
& E_{6}=\left\{\mathbf{u}: \mathbf{u} \in E_{8} ; \sum u_{i}=u_{7}+u_{8}=0\right\},
\end{aligned}
$$

and let $\Lambda_{24}$ denote the Leech lattice. In addition, we denote the dual lattice of $\Lambda$ by $\Lambda^{*}$. That is

$$
\Lambda^{*}=\left\{\mathbf{u} \in E^{n}:\langle\mathbf{u}, \mathbf{v}\rangle \in Z \text { for all } \mathbf{v} \in \Lambda\right\}
$$

where $\langle\mathbf{u}, \mathbf{v}\rangle$ indicates the inner product of $\mathbf{u}$ and $\mathbf{v}$. For every lattice $\Lambda$ there is a minimum number $\tau$ such that $B^{n}+\tau \Lambda$ is a packing. We list the known depths of the deep holes in some particular lattice ball packings in the following table. 
Table 3.1

\begin{tabular}{l|l|l}
$\Lambda$ & $\tau$ & $1+r\left(B^{n}, \tau \Lambda\right)$ \\
\hline$A_{n}$ & $\sqrt{2}$ & $\sqrt{\frac{2\left\lfloor\frac{n+1}{2}\right\rfloor\left(n+1-\left\lfloor\frac{n+1}{2}\right\rfloor\right)}{n+1}}$ \\
\hline$A_{n}^{*}$ & $2 \sqrt{(n+1) / n}$ & $\sqrt{(n+2) / 3}$ \\
\hline$D_{n}$ & $\sqrt{2}$ & $\begin{cases}\sqrt{2}, \quad n=3 \\
\sqrt{n / 2}, & n \geq 4\end{cases}$ \\
\hline & $\begin{cases}4 / \sqrt{3}, & n=3 \\
2, & n \geq 4\end{cases}$ & $\begin{cases}\sqrt{2 n-1}, 2, & n \text { odd } \geq 5 \\
\sqrt{n / 2}, & n \text { even }\end{cases}$ \\
\hline$D_{n}^{*}$ & $\sqrt{2}$ & $\sqrt{8 / 3}$ \\
\hline$E_{6}$ & $\sqrt{3}$ & $\sqrt{2}$ \\
\hline$E_{6}^{*}$ & $\sqrt{2}$ & $\sqrt{3}$ \\
\hline$E_{7}$ & $\sqrt{8 / 3}$ & $\sqrt{2}$ \\
\hline$E_{7}^{*}$ & $\sqrt{2}$ & $\sqrt{2}$ \\
\hline$E_{8}=E_{8}^{*}$ & 1 &
\end{tabular}

Although (2.1) provides a theoretical method to determine the deep holes and their depth in a particular lattice ball packing, it is still a hard job in practice, for example in $\Lambda_{24}, E_{6}^{*}$ and $E_{7}^{*}$ (see [7, 8], 35], [46] and [47]). Nevertheless, as one can predict, our knowledge about $\gamma^{*}\left(B^{n}\right)$ is more than that about $\gamma\left(B^{n}\right)$. Based on the methods and techniques developed by the Russian school, Horváth (see [26] and [27]) was able to determine the values of $\gamma^{*}\left(B^{4}\right)$ and $\gamma^{*}\left(B^{5}\right)$.

\section{Theorem 3.1.}

$$
\begin{aligned}
& \gamma^{*}\left(B^{4}\right)=\sqrt{2 \sqrt{3}}(\sqrt{3}-1), \\
& \gamma^{*}\left(B^{5}\right)=\sqrt{3 / 2+\sqrt{13} / 6} .
\end{aligned}
$$

Remark 2. It is well-known that every optimal packing lattice of the four-dimensional (five-dimensional) unit ball with respect to the density is isometric to $\sqrt{2} D_{4}\left(\sqrt{2} D_{5}\right)$, and every optimal covering lattice of the four-dimensional (five-dimensional) unit ball with respect to the density is isometric to $\sqrt{5 / 2} A_{4}^{*}\left(\sqrt{72 / 35} A_{5}^{*}\right)$. Clearly $r(C, X)$ can be regarded as a measure of efficiency of $X$ for the simultaneous packing and covering of $C$. Checking Table 3.1, it is remarkable to notice that the optimal lattice for the simultaneous lattice packing and lattice covering of $B^{4}$ is neither $D_{4}$ type nor $A_{4}^{*}$ type. The same is also true in $E^{5}$.

Remark 3. Comparing the value of $\gamma^{*}\left(B^{5}\right)$ with that of $1+r\left(B^{6}, E_{6}^{*}\right)$ (see Table 3.1), we get

$$
\gamma^{*}\left(B^{6}\right)<\gamma^{*}\left(B^{5}\right)
$$


which provides a negative answer to Problem 11.3 of Zong [51]. It is indeed very hard to imagine and to explain why this can happen.

When Rogers [39] studied the density of a ball packing he discovered a lower bound on the distance between a vertex and the center of a Dirichlet-Voronoi cell. In fact, by (2.1) his result implies

$$
\gamma\left(B^{n}\right) \geq \sqrt{\frac{2 n}{n+1}} .
$$

By this lower bound and some upper bounds provided by Table 3.1 we have

$$
\begin{aligned}
& \sqrt{12 / 7} \leq \gamma\left(B^{6}\right) \leq \gamma^{*}\left(B^{6}\right) \leq \sqrt{2}, \\
& \sqrt{7 / 4} \leq \gamma\left(B^{7}\right) \leq \gamma^{*}\left(B^{7}\right) \leq \sqrt{7 / 3}, \\
& \sqrt{16 / 9} \leq \gamma\left(B^{8}\right) \leq \gamma^{*}\left(B^{8}\right) \leq \sqrt{2}
\end{aligned}
$$

and

$$
\sqrt{1.92} \leq \gamma\left(B^{24}\right) \leq \gamma^{*}\left(B^{24}\right) \leq \sqrt{2} .
$$

Based on this observation it is reasonable to make the following conjecture.

\section{Conjecture 3.1.}

$$
\gamma\left(B^{8}\right)=\gamma^{*}\left(B^{8}\right)=\sqrt{2}
$$

and every optimal packing set is isometric to $\sqrt{2} E_{8}$.

$$
\gamma\left(B^{24}\right)=\gamma^{*}\left(B^{24}\right)=\sqrt{2}
$$

and every optimal packing set is isometric to $\Lambda_{24}$.

\section{Deep holes With Respect to the Minkowski metric}

While it is impossible to determine the values of $\gamma(C)$ and $\gamma^{*}(C)$ for all $n$ dimensional centrally symmetric convex bodies $C$, it is important to have some good bounds for them. Clearly, for every centrally symmetric convex body $C$ we have

$$
1 \leq \gamma(C)<2,
$$

where the lower bound can be attained only by tiles (the space filling bodies), and

$$
\gamma(C) \leq \gamma^{*}(C) .
$$

Let $\delta^{H}\left(C_{1}, C_{2}\right)$ denote the Hausdorff distance between $C_{1}$ and $C_{2}$. In other words,

$$
\delta^{H}\left(C_{1}, C_{2}\right)=\min \left\{r: r \geq 0 ; C_{1} \subseteq r B^{n}+C_{2} ; C_{2} \subseteq r B^{n}+C_{1}\right\} .
$$

If $C, C_{1}, C_{2}, \cdots$ is a sequence of $n$-dimensional centrally symmetric convex bodies satisfying

$$
\lim _{j \rightarrow \infty} \delta^{H}\left(C_{j}, C\right)=0
$$

then one can show both

$$
\gamma(C)=\lim _{j \rightarrow \infty} \gamma\left(C_{j}\right)
$$


and

$$
\gamma^{*}(C)=\lim _{j \rightarrow \infty} \gamma^{*}\left(C_{j}\right)
$$

In this section we will introduce some non-trivial bounds for $\gamma(C)$ and $\gamma^{*}(C)$. Let us start with the planar case.

Theorem 4.1. For every two-dimensional centrally symmetric convex domain $C$ we have

$$
\gamma(C)=\gamma^{*}(C) \leq 1.2 .
$$

As a consequence, intuitively speaking, for any considered domain $C$ there is a corresponding lattice packing $C+\Lambda$ in which one cannot add any translate of $\lambda C$ when $\lambda>0.2$.

In order to deduce the upper bound Zong [52] introduced two expressions for $\gamma^{*}(C)$, one in terms of an inscribed hexagon and another in terms of a regular triangle in the sense of the Minkowski metric $\|\cdot\|_{C}$. Then, by an argument involving continuous functions and mean value, one can apply the two expressions exactly in two cases and thus deduce the upper bound.

In fact, these expressions also provide practical methods to determine the exact value of $\gamma^{*}(C)$ for some particular domains. For example, letting $P_{n}$ denote the regular $n$-gon, one can show

$$
\gamma^{*}\left(P_{12 k}\right)=\gamma^{*}\left(B^{2}\right)=2 / \sqrt{3}=1.154700538 \cdots
$$

for all positive integers $k$ and especially

$$
\gamma^{*}\left(P_{8}\right)=\frac{4}{2+\sqrt{2}}=1.171572875 \cdots
$$

Remark 4. It is interesting to notice that

$$
\gamma^{*}\left(P_{8}\right)>\gamma^{*}\left(B^{2}\right)
$$

This means $B^{2}$ is not the least efficient domain with respect to the deep holes, though intuitively it should be. This phenomenon is very similar to the density problem (see Reinhardt [37] and Mahler [33]).

It is easy to check that the gap between $\gamma^{*}\left(P_{8}\right)$ and 1.2 is $0.028427124 \cdots$. However, since the upper bound is achieved by the mean value technique, we do not know the least efficient domains with respect to $\gamma^{*}(C)$.

Problem 4.1. What are the values of $\max \gamma(C)$ and $\max \gamma^{*}(C)$, where the maxima are over all $n$-dimensional centrally symmetric convex bodies $C$, and what are the extreme bodies?

To solve Problem 1.1 in three dimensions, Zong [53] obtained the following upper bound.

Theorem 4.2. For every three-dimensional centrally symmetric convex body $C$ we have

$$
\gamma^{*}(C) \leq 1.75
$$

Based on a classic result of Convex Geometry that every convex body can be arbitrarily approximated by smooth ones, we can assume that $C$ has a smooth surface. To prove the theorem, we start with a basic layer of translates of $C$ satisfying 
some particular technical conditions. Then we introduce a continuous function for all lattice arrangements of $C$ which consist of the translates of the basic layer. By varying the basic layer and changing the relative positions of the layers, we can find a lattice arrangement $C+\Lambda$ at which the function attains a special value. Then, by checking several cases determined by the function, we can prove that $C+\Lambda$ is a packing in $E^{3}$ and $1.75 C+\Lambda$ is a covering of $E^{3}$. Of course, some basic criteria for lattice packings and lattice coverings are essential in the proof. For example, let $\mathbf{a}_{1}, \mathbf{a}_{2}$ and $\mathbf{a}_{3}$ be a basis of a lattice $\Lambda$. If $C+\left\{\mathbf{o}, \mathbf{a}_{1}, \mathbf{a}_{2}, \mathbf{a}_{1}+\mathbf{a}_{2}, \mathbf{a}_{3}, \mathbf{a}_{1}+\mathbf{a}_{3}\right\}$ covers the convex hull of $\left\{\mathbf{o}, \mathbf{a}_{1}, \mathbf{a}_{2}, \mathbf{a}_{1}+\mathbf{a}_{2}, \mathbf{a}_{3}, \mathbf{a}_{1}+\mathbf{a}_{3}\right\}$, then $C+\Lambda$ is a covering of $E^{3}$.

It was observed by Minkowski that, for any convex set $A,(A+\mathbf{u}) \cap(A+\mathbf{v}) \neq \emptyset$ if and only if $\left(\frac{1}{2} D(A)+\mathbf{u}\right) \cap\left(\frac{1}{2} D(A)+\mathbf{v}\right) \neq \emptyset$, where $D(A)=\{\mathbf{x}-\mathbf{y}: \mathbf{x}, \mathbf{y} \in A\}$ is called the difference set of $A$. Since $D(A)$ is always centrally symmetric and convex, Theorem 4.2 has the following consequence.

Corollary 4.1. For any three-dimensional convex body $K$ there is a corresponding lattice packing $K+\Lambda$ in which no additional translate of $K$ can be added.

Now we turn to asymptotic bounds for $\gamma(C)$ and $\gamma^{*}(C)$ in high dimensions. Let $\theta(C)$ denote the density of the thinnest translative coverings of $E^{n}$ by translates of $C$ and let $\delta(C)$ denote the density of the densest translative packing of $C$ in $E^{n}$. For the lattice case, we will denote their analogues by $\theta^{*}(C)$ and $\delta^{*}(C)$ respectively. From the definitions of $\gamma(C), \theta(C)$ and $\delta(C)$ one can easily deduce

$$
\gamma(C) \geq \sqrt[n]{\frac{\theta(C)}{\delta(C)}} .
$$

It is well-known that

$$
\theta\left(B^{n}\right) \geq \tau_{n} \sim \frac{n}{e \sqrt{e}}
$$

and

$$
\delta\left(B^{n}\right) \leq 2^{-0.599 n(1+o(1))} .
$$

The lower bound for $\theta\left(B^{n}\right)$ was discovered by Coxeter, Few and Rogers [9], and the upper bound for $\delta\left(B^{n}\right)$ was deduced by Kabatjanski and Levenstein [29]. Thus we have the following lower bound for $\gamma\left(B^{n}\right)$.

\section{Theorem 4.3.}

$$
\gamma\left(B^{n}\right) \geq \sqrt[n]{\frac{\theta\left(B^{n}\right)}{\delta\left(B^{n}\right)}} \geq 2^{0.599}+o(1) .
$$

Remark 5. By Table 3.1 we have

$$
\gamma\left(B^{24}\right) \leq \gamma^{*}\left(B^{24}\right) \leq 1+r\left(B^{24}, \Lambda_{24}\right)=\sqrt{2} .
$$

It is interesting to notice that $\gamma^{*}\left(B^{24}\right)$ is smaller than $2^{0.599}$, the lower bound for $\gamma\left(B^{n}\right)$ when $n$ is sufficiently large.

Let $Q$ be a $k$-dimensional centrally symmetric convex body and let $Q^{\prime}$ be the $(k+1)$-dimensional cylinder with base $Q$ and height 2 . For a lattice arrangement $Q+\Lambda$ we write

$$
\sigma(Q, \Lambda)=\frac{v(Q)}{\operatorname{det}(\Lambda)}=\frac{v(Q)}{v(P)}
$$


and

$$
\epsilon(Q, \Lambda)=\frac{v(P \backslash\{Q+\Lambda\})}{v(P)},
$$

where $P$ indicates a fundamental parallelopiped of $\Lambda$. In 1959 , by a mean value method Rogers [40] proved that there is a $(k+1)$-dimensional lattice $\Lambda^{\prime}$ such that

$$
\sigma\left(Q^{\prime}, \Lambda^{\prime}\right)=2 \sigma(Q, \Lambda)
$$

and

$$
\epsilon\left(Q^{\prime}, \Lambda^{\prime}\right) \leq \epsilon(Q, \Lambda)^{2} .
$$

Then, by approximating the considered $n$-dimensional centrally symmetric convex body $C$ by generalized cylinders $Q \oplus I_{n-k}$ (here $I_{n-k}$ indicates a certain $(n-k)$ dimensional parallelopiped) and applying this result inductively, he was able to achieve the best known upper bound for $\theta^{*}(C)$.

Let $\chi(\mathbf{x})$ be the characteristic function of $2 C$. In 1945, Siegel [44 proved

$$
\int\left(\sum_{\mathbf{x} \in \Lambda \backslash\{\mathbf{o}\}} \chi(\mathbf{x})\right) d \sigma / \int d \sigma=v(2 C),
$$

where $d \sigma$ is the Haar measure over the space of all lattices with determinant 1. By this formula, one can immediately deduce the Minkowski-Hlawka theorem, the best known lower bound for $\delta^{*}(C)$.

Combining these two mean value techniques together, as a general upper bound for $\gamma^{*}(C)$ in higher dimensions, Butler [6] proved the following surprising result.

Theorem 4.4. When $n$ is sufficiently large,

$$
\gamma^{*}(C) \leq 2+o(1)
$$

holds for every $n$-dimensional centrally symmetric convex body $C$.

Comparing with Problem 1.1, one can immediately understand the significance of this theorem. Although this theorem cannot solve the problem, the $o(1)$ term makes the problem mysterious.

Remark 6. Before Butler's work, Rogers [38] proved that if $C+\Lambda$ is a lattice packing with

$$
1+r(C, \Lambda) \geq 3
$$

then one can construct a new lattice packing $C+\Lambda^{\prime}$ such that $\Lambda \subset \Lambda^{\prime}$ and

$$
\operatorname{det}\left(\Lambda^{\prime}\right)=\operatorname{det}(\Lambda) / 3 .
$$

Therefore we have

$$
\gamma^{*}(C)<3 .
$$

For the ball case, by modifying Rogers' method, Henk [18] improved the upper bound from 3 to $\sqrt{21} / 2$. 


\section{INFINITE CYLINDRICAL HOLES IN LATTICE BALL PACKINGS}

In 1960 Heppes [22] discovered the following remarkable fact:

In every three-dimensional lattice ball packing there is a straight line of infinite length which does not intersect any of the balls.

In fact his proof implied that in every lattice ball packing $B^{3}+\Lambda$ there is a cylinder $O$ of infinite length (in both directions) such that

$$
O \cap\left\{\operatorname{int}\left(B^{3}\right)+\Lambda\right\}=\emptyset .
$$

We call such a cylinder a cylindrical hole in the lattice ball packing.

Let $r_{1}^{*}\left(B^{n}\right)$ be the maximum number such that in every $n$-dimensional lattice ball packing $B^{n}+\Lambda$ there is a cylindrical hole with an $(n-1)$-dimensional spherical base of radius $r_{1}^{*}\left(B^{n}\right)$. To improve Heppes' result, Hortobágyi [24] was able to determine the value of $r_{1}^{*}\left(B^{3}\right)$. As one can imagine, his proof was very technical and complicated.

\section{Theorem 5.1.}

$$
r_{1}^{*}\left(B^{3}\right)=\frac{3 \sqrt{2}}{4}-1=0.060660171 \cdots
$$

In addition, $\sqrt{2} A_{3}$ is the only corresponding optimal lattice with respect to isometry.

To study the cylindrical holes in lattice ball packings, Horváth 25] discovered a basic concept and a fundamental lemma about lattices. Let $H\left(\mathbf{x}_{1}, \mathbf{x}_{2}, \cdots, \mathbf{x}_{m}\right)$ denote the $(m-1)$-dimensional plane determined by $\mathbf{x}_{1}, \mathbf{x}_{2}, \cdots, \mathbf{x}_{m}$. A Seeber set of an $n$-dimensional lattice $\Lambda$ is a set of $n$ points $\left\{\mathbf{u}_{1}, \mathbf{u}_{2}, \cdots, \mathbf{u}_{n}\right\}$ such that, for $i=1,2, \cdots, n, \mathbf{u}_{i}$ is a point of $\Lambda \backslash H\left(\mathbf{o}, \mathbf{u}_{i-1}, \mathbf{u}_{i-2}, \cdots\right)$ with minimum norm. Then Horváth's lemma can be stated as follows.

Lemma 5.1. Let $\Lambda$ be a packing lattice of $B^{n}$ and let $\left\{\mathbf{u}_{1}, \mathbf{u}_{2}, \cdots, \mathbf{u}_{n}\right\}$ be a Seeber set of $\Lambda$. Denote by $H_{i}$ the hyperplane $\left\{\mathbf{x}:\left\langle\mathbf{x}, \mathbf{u}_{i}\right\rangle=0\right\}$ and by $p_{i}(\mathbf{x})$ the projection of $\mathbf{x}$ onto $H_{i}$. Then

$$
\left\|\mathbf{o}, p_{i}(\mathbf{u})\right\| \geq \sqrt{3}
$$

holds for every point $\mathbf{u} \in \Lambda \backslash H\left(\mathbf{o}, \mathbf{u}_{i}\right)$.

By this lemma, the cylindrical holes in direction $\mathbf{u}_{i}$ are projected into spherical holes in $B^{n-1}+p_{i}(\Lambda)$. Lemma 5.1 is not powerful enough to provide an elegant proof for Theorem 5.1. However, it is very useful in deducing the following lower bound for $r_{1}^{*}\left(B^{n}\right)$ (see Ryskov and Horváth [43]).

\section{Theorem 5.2.}

$$
r_{1}^{*}\left(B^{n}\right) \geq \frac{\sqrt{3}}{2} \gamma^{*}\left(B^{n-1}\right)-1 \geq 0.3117395+o(1)
$$

When $n=4$, it follows by this theorem that

$$
r_{1}^{*}\left(B^{4}\right) \geq \frac{\sqrt{3}}{2} \cdot \sqrt{\frac{5}{3}}-1=\frac{\sqrt{5}}{2}-1 .
$$

On the other hand, by applying Lemma 5.1 to $\sqrt{2} D_{4}$, in $B^{4}+\sqrt{2} D_{4}$ one can find cylindrical holes with spherical bases of radius exactly $\sqrt{5} / 2-1$. Therefore we have the following result discovered by Horváth [25]. 
Theorem 5.3.

$$
r_{1}^{*}\left(B^{4}\right)=\frac{\sqrt{5}}{2}-1=0.118033988 \cdots
$$

and $B^{4}+\sqrt{2} D_{4}$ is a corresponding extreme packing.

It follows by Lemma 5.1 that every Seeber set provides $n$ directions in which one can find cylindrical holes. On the other hand, every shortest vector of a lattice belongs to some Seeber set. It is well-known that the Leech lattice has 196560 shortest vectors. Thus, in $B^{24}+\Lambda_{24}$ there are at least 196560/2 different directions in which one can find cylindrical holes with spherical bases of radius not smaller than $\sqrt{23} / 4-1=0.19895788 \cdots$.

\section{Free segments in BALl PACKings}

It is natural to ask: Is there a similar infinite cylindrical hole in every translative ball packing? As will be shown at the end of this section, the answer is "no". Before dealing with this problem, let us first introduce some related local results which have their own significance.

For convenience, we call a straight line with one side to infinity a light ray and say $C+\mathbf{x}$ and $C+\mathbf{y}$ are nonoverlapping if $(\operatorname{int}(C)+\mathbf{x}) \cap(\operatorname{int}(C)+\mathbf{y})=\emptyset$. Let $\psi(C)$ denote the smallest number of nonoverlapping translates of $C$, all of them having no common point with $\operatorname{int}(C)$, which can block all the light rays starting from the center of $C$. Let $\varphi(C)$ denote the smallest number of nonoverlapping translates of $C$, all of them having no common point with $\operatorname{int}(C)$, which can block all the light rays starting from $C$. In addition, let $\psi^{*}(C)$ and $\varphi^{*}(C)$ denote their lattice analogues (the translate points form a subset of a lattice), respectively. By the definitions we have

$$
\psi(C) \leq \psi^{*}(C)
$$

and

$$
\psi(C) \leq \varphi(C) \leq \varphi^{*}(C) .
$$

In the planar case, since we need exactly six circles to surround one, we can deduce

$$
\psi\left(B^{2}\right)=\psi^{*}\left(B^{2}\right)=\varphi\left(B^{2}\right)=\varphi^{*}\left(B^{2}\right)=6 .
$$

In fact, this is true for all two-dimensional centrally symmetric convex domains except parallelograms. For the exceptions the answer is four.

It follows from Theorem 5.1 and Theorem 5.2 that in every $n$-dimensional lattice ball packing $(n \geq 3)$ there exist cylindrical holes. Therefore we have

$$
\varphi^{*}\left(B^{n}\right)=\infty
$$

for $n \geq 3$. As one can predict, when $n \geq 3$, determining the values of $\psi\left(B^{n}\right)$, $\psi^{*}\left(B^{n}\right)$ and $\varphi\left(B^{n}\right)$ is a very challenging job.

According to L. Fejes Tóth 14 it was Hornich who first proposed the problem of determining $\psi\left(B^{3}\right)$. Through the works of L. Fejes Tóth, Heppes, Csóka, Danzer, etc., we now have the following bounds for $\psi\left(B^{3}\right)$.

\section{Theorem 6.1.}

$$
30 \leq \psi\left(B^{3}\right) \leq 42 .
$$


Turning to higher dimensions, we have the following asymptotic bounds for $\psi(C)$ and $\psi^{*}(C)$.

Theorem 6.2. When $n$ is sufficiently large,

$$
\psi^{*}(C) \leq \frac{\delta(C)^{n}}{\delta^{*}(C)^{n}} 2^{n^{2}(1+o(1))}
$$

holds for every $n$-dimensional centrally symmetric convex body $C$.

Theorem 6.3.

$$
2^{0.275 n^{2}(1+o(1))} \leq \psi\left(B^{n}\right) \leq \psi^{*}\left(B^{n}\right) \leq 2^{1.401 n^{2}(1+o(1))} .
$$

In 1999, Zong [50] discovered a close relationship between $\psi^{*}(C)$ and Dirichlet's approximation theorem in Number Theory from which he deduced the first general upper bound for $\psi^{*}(C)$. Then Henk (see [51]) provided the following geometric version of Dirichlet's theorem from which one can deduce Theorem 6.2.

Lemma 6.1. Let $C+\Lambda$ be a lattice packing in $E^{n}$ with density $\delta(C, \Lambda)$. For any vector $\mathbf{v} \neq \mathbf{o}$ there is a lattice point $\mathbf{u}$ and a positive integer

$$
z \leq \frac{2^{n} \delta(C)}{\delta(C, \Lambda)}
$$

such that

$$
z \mathbf{v} \in \operatorname{int}(C)+\mathbf{u} .
$$

It is well-known that

$$
\delta\left(B^{n}\right) \leq 2^{-0.599 n(1+o(1))}
$$

and

$$
\delta^{*}\left(B^{n}\right) \geq \frac{\zeta(n)}{2^{n-1}},
$$

where $\zeta(x)$ is the Riemann zeta-function (see [16] or [51]). Therefore one can easily deduce the upper bound in Theorem 6.3 from Theorem 6.2.

For convenience, we write $r=2^{0.275 n}, \alpha=1 / n$ and $r_{i}=2+i \alpha$. Let $X$ be a packing set of $B^{n}$ such that $B^{n}+X$ does block all the light rays starting from $\mathbf{o}$, and write

$$
X_{i}=\left\{\mathbf{x} \in X: r_{i} \leq\|\mathbf{x}\|<r_{i+1}\right\} .
$$

By known results about cap packing, one can deduce

$$
\operatorname{card}\left\{X_{i}\right\} \leq r^{n} 2^{-0.552 n(1+o(1))} .
$$

Then, by comparing the spherical areas, one can prove that the spherical projections of those $B^{n}+\mathbf{x}, \mathbf{x} \in X \cap r B^{n}$, onto the surface $\partial\left(B^{n}\right)$ of $B^{n}$ cannot cover half of it when $n$ is sufficiently large. Thus, to cover the left part of $\partial\left(B^{n}\right)$ by the corresponding spherical projections of the unit balls with centers outside $r B^{n}$, Bárány and Leader (see [51]) were able to get the lower bound in Theorem 6.3. A similar lower bound can also be found in Talata [45.

In 1959, L. Fejes Tóth [14] proposed the problem to determine $\varphi\left(B^{3}\right)$. Although it seems more natural than $\psi\left(B^{3}\right)$, our knowledge about $\varphi\left(B^{3}\right)$ is even less. So far, we only know

$$
30 \leq \varphi\left(B^{3}\right) \leq 326
$$


where the upper bound was discovered by Schopp (see 4]).

In higher dimensions we have the following upper bound for $\varphi(C)$.

Theorem 6.4. When $n$ is sufficiently large,

$$
\varphi(C) \leq 3^{n^{2}(1+o(1))}
$$

holds for every $n$-dimensional centrally symmetric convex body $C$.

This theorem was proved by the following technique: First, we construct a suitable covering on the surface of $r C$ with translates of $\alpha C$, where $r$ is a large number and $\alpha$ is a small positive number which will be chosen later. Second, enlarge the translates to $C$ and remove them along their radical directions to suitable positions to form a packing. Third, applying some argument based on doublecone, make sure that the new packing does block all the light rays starting from $C$. Finally, by choosing suitable parameters $r$ and $\alpha$, compute the number of the translates to get the upper bound. This technique was first discovered by Zong [49]. Later, improvements were made by Talata [45]. Böröczky and Soltan [4] obtained a weaker upper bound simultaneously and independently. In fact, they proved that

$$
\varphi(C) \leq c_{n}
$$

holds for a suitable constant $c_{n}$ depending only on $n$.

Based on the following observation, one can deduce Theorem 6.4 also from Lemma 6.1.

If $C+\mathbf{x}$ blocks the light ray starting from $\mathbf{o}$ and in the direction $\mathbf{u}$, then $2 C+\mathbf{x}$ will block all the light rays starting from $C$ and in the direction

u.

It was Bárány who first made such an observation and deduced Theorem 6.4 from Lemma 6.1 for the ball case. Then it was generalized to arbitrary centrally symmetric convex bodies by the author (see 51]).

For the ball case, by (6.1) and Theorems 6.3 and 6.4 , one can easily deduce

$$
2^{0.275 n^{2}(1+o(1))} \leq \varphi\left(B^{n}\right) \leq 3^{n^{2}(1+o(1))} .
$$

To tighten these bounds Talata [45] was able to show the following result.

\section{Theorem 6.5.}

$$
2^{0.599 n^{2}(1+o(1))} \leq \varphi\left(B^{n}\right) \leq 2^{1.401 n^{2}(1+o(1))} .
$$

Based on Theorem 6.3 and Theorem 6.5, Zong [51] proposed two problems about the asymptotic orders of $\psi\left(B^{n}\right), \psi^{*}\left(B^{n}\right)$ and $\varphi\left(B^{n}\right)$. We give them here.

Problem 6.1. Do there exist absolute constants $c$ and $c^{\prime}$ such that

$$
\psi\left(B^{n}\right)=2^{c n^{2}(1+o(1))}
$$

or

$$
\psi^{*}\left(B^{n}\right)=2^{c^{\prime} n^{2}(1+o(1))} ?
$$

If so, determine them.

Problem 6.2. Does there exist an absolute constant $c^{*}$ such that

$$
\varphi\left(B^{n}\right)=2^{c^{*} n^{2}(1+o(1))} ?
$$

If so, determine it. 
Besides their own significance, Theorems $6.1-6.5$ provide a local picture about segments in packings. Let $s(C, X)$ denote the maximum length (in the Minkowski metric $\|\cdot\|_{C}$ ) of the segments contained in $E^{n} \backslash\{\operatorname{int}(C)+X\}$ and define

$$
s(C)=\min _{X} s(C, X),
$$

where the minimum is over all packing sets of $C$. To answer the question stated at the beginning of this section, based on the periodic property of a lattice, Henk and Zong [20] were able to prove the following result by induction.

Theorem 6.6. For any fixed $\epsilon, \epsilon>0$, there exist a periodic set $X(n, \epsilon)$ and a constant $c(n, \epsilon)$ such that $B^{n}+X(n, \epsilon)$ is a packing in $E^{n}$ and

$$
s\left(\epsilon B^{n}, X(n, \epsilon)\right) \leq c(n, \epsilon) .
$$

Corollary 6.1. For every convex body $K$, even if not centrally symmetric, we have

$$
\kappa(K)=0 \text {. }
$$

Remark 7. This result not only provides a negative answer to the question stated at the beginning of this section, it also shows an essential difference between lattice packings and nonlattice packings.

It is well-known that for every $n$-dimensional centrally symmetric convex body $C$ there is a linear transformation $L$ such that

$$
B^{n} \subseteq L(C) \subseteq \sqrt{n} B^{n}
$$

(see [48]). Therefore, by Theorem 6.6 one can deduce

$$
s(C) \leq c(n),
$$

where $c(n)$ is a constant depending only on $n$. To improve this bound, Böröczky and Tardo [5] show the following result with a probability method.

Theorem 6.7. When $n$ is sufficiently large,

$$
s(C) \leq 2^{n(1+o(1))}
$$

holds for every $n$-dimensional centrally symmetric convex body $C$.

For convenience, we write $\beta=10000$ and assume $n>2$. By the MinkowskiHlawka theorem (see [16] or [51]), for the considered $C$ there is a lattice $\Lambda$ such that $4 \beta n^{2} 4^{n} C+\Lambda$ is a packing and

$$
\operatorname{det}(\Lambda) \leq 2 \beta n^{2} 4^{2 n} v(C) .
$$

Let $T$ denote the torus $R^{n} / \Lambda$ and throw points $\mathbf{x}_{1}, \mathbf{x}_{2}, \cdots$ randomly into $T$ with uniform distribution. We will color $\mathbf{x}_{i}$ in green if $C+\mathbf{x}_{i}$ is disjoint with any $C+\mathbf{x}_{j}$ for $j<i$. For a measurable subset $A$ of $T$ we denote the possibility that $A$ contains no green point by $p(A)$. According to Rogers [41] there is a covering $\frac{1}{n} C+Y$ of $T$ with a density at most $n \log n+n \log \log n+5 n$. Therefore we have

$$
\operatorname{card}\{Y\} \leq(n \log n+n \log \log n+5 n) n^{n} \frac{\operatorname{det}(\Lambda)}{v(C)} \leq 2^{10 n^{2}} .
$$

Let $\bar{S}$ denote the family of the segments in $T$ with Minkowski length between $\beta n^{2} 2^{n}-1$ and $\beta n^{2} 2^{n}+1$ and with endpoints belonging to $Y$. Clearly we have

$$
\operatorname{card}\{\bar{S}\} \leq \operatorname{card}\{Y\}^{2} \leq 2^{20 n^{2}}
$$


Writing $N=\left\lfloor\frac{\beta}{6} n^{2} 2^{n}\right\rfloor$, one can prove that

$$
p((1-2 / n) C+S) \leq\left(1-(1 / 2-1 / n)^{n}\right)^{N}
$$

holds for every segment $S \in \bar{S}$. Thus, the possibility $p_{0}$ that there exists an $S \in \bar{S}$ such that $(1-2 / n) C+S$ contains no green point satisfies

$$
p_{0} \leq \operatorname{card}\{\bar{S}\} \cdot p((1-2 / n) C+S)<1 .
$$

Therefore there is a sequence of points $\mathbf{x}_{1}, \mathbf{x}_{2}, \cdots$ such that, for any $S \in \bar{S}$, the set $(1-2 / n) C+S$ contains a green point. Let $X$ denote the set of the green points. It can be proved that $C+X+\Lambda$ is a periodic packing in which no free segment is longer than $\beta n^{2} 2^{n}$.

By the lower bound of $\varphi\left(B^{n}\right)$ in Theorem 6.5 one can deduce

$$
s\left(B^{n}\right) \geq 2^{0.599 n(1+o(1))} .
$$

In fact, this lower bound can also be easily deduced from a random argument based on the known upper bound for $\delta\left(B^{n}\right)$ (see [5]). This lower bound and Theorem 6.7 support a positive answer for the following problem proposed by Zong [51].

Problem 6.3. Does there exist an absolute constant $c$ such that

$$
s\left(B^{n}\right)=2^{c n(1+o(1))} ?
$$

If so, determine it.

\section{From DEEP HOLES TO FREE PLANES}

Let $\mu_{i}(C, \Lambda)$ denote the minimum number $\lambda$ such that $E^{n} \backslash\{\lambda C+\Lambda\}$ does not contain any $(n-i)$-dimensional plane. Usually, $\mu_{i}(C, \Lambda)$ is called the $i$-th covering minimum of $C$ with respect to $\Lambda$. This sequence of numbers was introduced by Kannan and Lovász [30] in 1988. Clearly we have

$$
\mu_{1}(C, \Lambda) \leq \mu_{2}(C, \Lambda) \leq \cdots \leq \mu_{n}(C, \Lambda) .
$$

In some sense, covering minima can be regarded as a counterpart to Minkowski's successive minima (see [16) in Geometry of Numbers.

In fact, it is these covering minima which join deep holes, cylindrical holes and free planes together. Now, let us explain the connections.

First, it is easy to see that $\mu_{n}(C, \Lambda)$ is nothing else but the depth of the deep holes in $C+\Lambda$ with respect to the Minkowski metric $\|\cdot\|_{C}$ when $C+\Lambda$ is a packing. Therefore we have

$$
\gamma^{*}(C)=\min _{\Lambda} \mu_{n}(C, \Lambda),
$$

where the minimum is over all the packing lattices of $C$. For a general lattice arrangement $C+\Lambda$, let $\eta(C, \Lambda)$ denote the maximum number $\eta$ such that $\eta C+\Lambda$ is a packing. Then one can deduce

$$
\gamma^{*}(C)=\min _{\Lambda} \frac{\mu_{n}(C, \Lambda)}{\eta(C, \Lambda)},
$$

where the minimum is over all lattices. Usually, we call $\mu_{n}(C, \Lambda)$ the covering radius of $C$ with respect to $\Lambda$ and call $\eta(C, \Lambda)$ the packing radius of $C$ with respect to $\Lambda$. In fact, $2 \eta(C, \Lambda)$ is nothing else but the first successive minimum of $C$ with respect to $\Lambda$ (see [16]). 
Second, it follows from their definitions that

$$
\mu_{n-\kappa^{*}(C)}(C, \Lambda) \geq \eta(C, \Lambda)
$$

holds for every lattice. In other words, $\kappa^{*}(C)$ is the largest integer such that the above inequality holds for all lattices.

Third, we define

$$
r_{i}^{*}(C)=\min _{\Lambda}\left\{\mu_{n-i}(C, \Lambda)-1\right\},
$$

where the minimum is over all the packing lattices of $C$. When $i \leq \kappa^{*}(C)$ we have

$$
r_{i}^{*}(C) \geq 0 .
$$

Thus in every lattice packing $C+\Lambda$ there is a corresponding $i$-dimensional plane $F_{i}$ such that

$$
r_{i}^{*}(C) C+F_{i} \subset E^{n} \backslash\{\operatorname{int}(C)+\Lambda\} .
$$

Let $C, C_{1}, C_{2}, \cdots$ be a sequence of $n$-dimensional centrally symmetric convex bodies satisfying

$$
\lim _{j \rightarrow \infty} \delta^{H}\left(C_{j}, C\right)=0 .
$$

One can deduce

$$
\kappa^{*}(C) \geq \limsup _{j \rightarrow \infty} \kappa^{*}\left(C_{j}\right)
$$

and

$$
r_{i}^{*}(C)=\lim _{j \rightarrow \infty} r_{i}^{*}\left(C_{j}\right)
$$

for all $i \leq n$. However, we do not know the answer to the following problem.

Problem 7.1. Is there a sequence of $n$-dimensional centrally symmetric convex bodies $C, C_{1}, C_{2}, \cdots$ satisfying both

$$
\lim _{j \rightarrow \infty} \delta^{H}\left(C_{j}, C\right)=0
$$

and

$$
\limsup _{j \rightarrow \infty} \kappa^{*}\left(C_{j}\right) \neq \liminf _{j \rightarrow \infty} \kappa^{*}\left(C_{j}\right) ?
$$

Remark 8. Clearly the number sequence $r_{i}^{*}(C), 0 \leq i \leq \kappa^{*}(C)$, provides an intuitive measure for the efficiency of lattice packings. Determining the asymptotic order of $\delta^{*}\left(B^{n}\right)$ is one of the most important problems in Packing and Covering. However, the gap between its known lower bound, $2^{-n(1+o(1))}$ (the MinkowskiHlawka theorem), and its known upper bound, $2^{-0.599 n(1+o(1))}$ (the KabatjanskiLevenstein theorem), is still huge. Better understanding of the sequence $r_{i}^{*}\left(B^{n}\right)$ perhaps will bring progress to this problem.

It is easy to see that

$$
\gamma^{*}(C)=1+r_{0}^{*}(C) .
$$

Therefore one can ask questions similar to Problem 4.1 for $r_{i}^{*}(C)$. Based on Remark 4 it seems that $B^{n}$ cannot be an extreme body for questions of this kind. Nevertheless, since $B^{n}$ is the most typical centrally symmetric convex body in $E^{n}$, we end this section with the following problem. 
Problem 7.2. Is it true that

$$
\kappa^{*}\left(B^{n}\right) \geq \kappa^{*}(C)
$$

holds for every $n$-dimensional centrally symmetric convex body $C$ ?

\section{Free Planes in LATtice BALL PACKINGS}

In 1970, when he discovered the Seeber set and applied it to the study of cylindrical holes, Horváth [25] claimed a proof for the following statement:

In every $n$-dimensional lattice ball packing there exists an $(n-2)$ -

dimensional free plane.

In other words,

$$
\kappa^{*}\left(B^{n}\right) \geq n-2 .
$$

However, in 1992 Hausel [17] found a mistake in Horváth's proof and showed that

$$
\kappa^{*}\left(B^{n}\right) \leq n-c \sqrt{n}
$$

for some suitable positive constant $c$. Recently, Henk, Ziegler and Zong [21] improved this upper bound to the following result.

Theorem 8.1. There is an absolute constant $c, 0<c<1$, such that

$$
\kappa^{*}\left(B^{n}\right) \leq c n .
$$

It was shown by Kannan and Lovász [30] that

$$
2 \mu_{1}\left(B^{n}, \Lambda\right)=1 / 2 \eta\left(B^{n}, \Lambda^{*}\right)
$$

holds for every $n$-dimensional lattice. In addition, Conway and Thompson (see [34]) proved that there exists a constant $\beta_{1}$ such that for every dimension $n$ one can find a lattice $\Lambda$ satisfying

$$
\eta\left(B^{n}, \Lambda\right) \cdot \eta\left(B^{n}, \Lambda^{*}\right) \geq \beta_{1} n .
$$

Thus, for those lattices we have

$$
\frac{\mu_{1}\left(B^{n}, \Lambda\right)}{\eta\left(B^{n}, \Lambda\right)} \leq \frac{1}{4 \beta_{1} n} .
$$

On the other hand, it was proved by Banaszczyk 2] that there is a positive constant $\beta_{2}$ such that

$$
\mu_{n}\left(B^{n}, \Lambda\right) \leq \beta_{2} n \mu_{1}\left(B^{n}, \Lambda\right)
$$

holds for every $n$-dimensional lattice. In addition, for any fixed $n$-dimensional lattice $\Lambda$ and $k \in\{1,2, \cdots, n\}$, one can construct a $k$-dimensional lattice $\Lambda_{k}$ satisfying both

$$
\mu_{k}\left(B^{n}, \Lambda\right)=\mu_{k}\left(B^{k}, \Lambda_{k}\right)
$$

and

$$
\mu_{1}\left(B^{n}, \Lambda_{k}\right) \leq \mu_{1}\left(B^{n}, \Lambda\right) .
$$

Then, for a lattice satisfying (8.1) we have

$$
\frac{\mu_{k}\left(B^{n}, \Lambda\right)}{\eta\left(B^{n}, \Lambda\right)}=\frac{\mu_{k}\left(B^{k}, \Lambda_{k}\right)}{\eta\left(B^{n}, \Lambda\right)} \leq \frac{\beta_{2} k \mu_{1}\left(B^{n}, \Lambda\right)}{\eta\left(B^{n}, \Lambda\right)} \leq \frac{\beta_{2} k}{4 \beta_{1} n} .
$$


Therefore, for $k<\frac{4 \beta_{1}}{\beta_{2}} n$, the lattice ball packing $\eta\left(B^{n}, \Lambda\right) B^{n}+\Lambda$ does not contain any $(n-k)$-dimensional free plane.

In fact, Horváth's method fails even in proving $\kappa^{*}\left(B^{n}\right) \geq 2$. Before presenting a lower bound for $\kappa^{*}\left(B^{n}\right)$, let us introduce a couple of examples.

Example 8.1. Define an $(n-2)$-dimensional plane

$$
F_{n-2}=\left\{\left(\sqrt{2} / 2, \sqrt{2} / 2, x_{3}, \cdots, x_{n}\right): x_{i} \in R\right\}
$$

in $E^{n}$. For any $\mathbf{x} \in F_{n-2}$ and $\mathbf{u}=\sqrt{2} \mathbf{z} \in \sqrt{2} D_{n}$ we have

$$
\|\mathbf{x}, \mathbf{u}\| \geq \sqrt{2\left[\left(0.5-z_{1}\right)^{2}+\left(0.5-z_{2}\right)^{2}\right]} \geq 1 .
$$

This means that $F_{n-2}$ is an $(n-2)$-dimensional free plane in $B^{n}+\sqrt{2} D_{n}$. A similar statement is also true for $\sqrt{2} A_{n}$.

Example 8.2. It is known that $B^{8}+\sqrt{2} E_{8}$ is the densest lattice ball packing in $E^{8}$. Define a four-dimensional plane

$$
F_{4}=\left\{\left(\sqrt{2} / 2, \sqrt{2} / 2,0,0, x_{5}, \cdots, x_{8}\right): x_{i} \in R\right\}
$$

in $E^{8}$. For any $\mathbf{x} \in F_{4}$ and $\mathbf{u} \in \sqrt{2} E_{8}$ we have

$$
\|\mathbf{x}, \mathbf{u}\| \geq \sqrt{2\left(0.5^{2}+0.5^{2}\right)}=1 \text {. }
$$

This means that $F_{4}$ is a four-dimensional free plane in $B^{8}+\sqrt{2} E_{8}$. Similarly, one can find three-dimensional free planes in both $B^{7}+\sqrt{2} E_{7}$ and $B^{6}+\sqrt{2} E_{6}$.

Clearly, determining the maximum dimension of the free planes in a particular lattice packing is an interesting and non-trivial problem. Here we emphasize the following one.

Problem 8.1. Determine the maximum dimension of the free planes in $B^{24}+\Lambda_{24}$.

As for a lower bound for $\kappa^{*}\left(B^{n}\right)$ we have the following result.

Theorem 8.2. For any fixed $\epsilon>0$,

$$
\kappa^{*}\left(B^{n}\right) \geq \begin{cases}2, & \text { if } n \geq 5, \\ 3, & \text { if } n=8, \\ n^{1-\epsilon}, & \text { if } n \text { is sufficiently large. }\end{cases}
$$

The first two cases of this theorem were proved by Henk, Ziegler and Zong [21] by applying Korkin-Zolotarev reduction in quadratic forms and some known results about the deep holes introduced in previous sections. The third case was proved by Henk [19] by applying generalized Hermite constants.

Let $\Lambda_{k}$ denote a $k$-dimensional sublattice of $\Lambda$ of minimum determinant and define

$$
\bar{\gamma}(n, k)=\max _{\Lambda} \frac{\operatorname{det}\left(\Lambda_{k}\right)^{1 / k}}{\operatorname{det}(\Lambda)^{1 / n}}
$$

where the maximum is over all $n$-dimensional lattices $\Lambda$. Usually the $n$ numbers $\bar{\gamma}(n, k)^{2 k}$ for $k=1,2, \cdots, n$ are called generalized Hermite constants. They were first studied by Rankin [36]. By the definitions one can prove

$$
\frac{\mu_{k}\left(B^{n}, \Lambda\right)}{2 \eta\left(B^{n}, \Lambda\right)} \geq \frac{1}{\bar{\gamma}(n, 1) \bar{\gamma}(n, k) \omega_{k}^{1 / k}}
$$


where $\omega_{k}$ indicates the volume of the $k$-dimensional unit ball. On the other hand we have

$$
c_{1} \sqrt{n} \leq \bar{\gamma}(n, 1) \leq c_{2} \sqrt{n}
$$

and

$$
\bar{\gamma}(n, k) \leq\left(c_{3} \sqrt{k+1}\right)^{\frac{n-k}{k}}
$$

for some suitable constants $c_{1}, c_{2}$ and $c_{3}$. Thus we can deduce the third case of Theorem 8.2 from (8.2).

By Theorem 8.1 and Theorem 8.2, for sufficiently large $n$, we have

$$
n^{1-\epsilon} \leq \kappa^{*}\left(B^{n}\right) \leq c n
$$

for any fixed $\epsilon>0$ and some suitable $c<1$. Thus we end this paper with the following modified version of Problem 1.3.

Problem 1.3'. Does there exist a positive constant $c$ such that

$$
\lim _{n \rightarrow \infty} \frac{\kappa^{*}\left(B^{n}\right)}{n}=c ?
$$

If so, determine it.

\section{ACKNowledgements}

I am very grateful to E. Bannai, W. Y. Ding, P. M. Gruber, M. Z. Guo, M. Henk, D. G. Larman, D. G. Saari, A. Schürmann, D. Wang, J. P. Zhang and the referees for their helpful remarks, suggestions and comments, and to Ms. D. Smith at the AMS for her editorial help.

\section{REFERENCES}

[1] R. P. Bambah, On lattice coverings by spheres, Proc. Nat. Inst. Sci. India 20 (1954), 25-52. MR 15:780c

[2] W. Banaszczyk, New bounds in some transference theorems in the geometry of numbers, Math. Ann. 296 (1993), 625-636. MR 94k:11075

[3] K. Böröczky, Closest packing and loosest covering of the space with balls, Studia Sci. Math. Hungar. 21 (1986), 79-89. MR 88g:52003

[4] K. Böröczky and V. Soltan, Translational and homothetic clouds for a convex body, Studia Sci. Math. Hungar. 32 (1996), 93-102. MR 97h:52008

[5] K. Böröczky Jr. and G. Tardos, The longest segment in the complement of a packing, Mathematika, in press.

[6] G. J. Butler, Simultaneous packing and covering in Euclidean space, Proc. London Math. Soc. 25 (1972), 721-735. MR 47:7600

[7] J. H. Conway, R. A. Parker and N. J. A. Sloane, The covering radius of the Leech lattice, Proc. Royal Soc. London A 380 (1982), 261-290. MR 84m:10022b

[8] J. H. Conway and N. J. A. Sloane, Sphere Packings, Lattices and Groups (third edition), Springer-Verlag, New York, 1999. MR 2000b:11077

[9] H. S. M. Coxeter, L. Few and C. A. Rogers, Covering space with equal spheres, Mathematika 6 (1959), 147-157. MR 23:A2131

[10] G. Csóka, The number of congruent spheres that hide a given sphere of three-dimensional space is not less than 30, Studia Sci. Math. Hungar. 12 (1977), 323-334. MR 82m:52008

[11] L. Danzer, Drei Beispiele zu Lagerungsproblemen, Arch. Math. 11 (1960), 159-165.

[12] B. N. Delone and S. S. Ryskov, Solution of the problem of least dense lattice covering of a four-dimensional space by equal spheres, Soviet Math. Dokl. 4 (1963), 1333-1334; Dokl. Acad. Nauk SSSR 152 (1963), 523-524. MR 31:126

[13] G. Fejes Tóth and W. Kuperberg, Packing and Covering with Convex Sets, Handbook of Convex Geometry (eds. P. M. Gruber and J. M. Wills), North-Holland, Amsterdam (1993), 799-860. MR 95d:52020 
[14] L. Fejes Tóth, Verdeckung einer Kugel durch Kugeln, Publ. Math. Debrecen 6 (1959), 234240. MR 22:4017

[15] L. Fejes Tóth, Close packing and loose covering with balls, Publ. Math. Debrecen 23 (1976), 323-326. MR 55:1224

[16] P. M. Gruber and C. G. Lekkerkerker, Geometry of Numbers (2nd ed.), North-Holland, Amsterdam, 1987. MR 88j:11034

[17] T. Hausel, Transillumination of lattice packing of balls, Studia Sci. Math. Hungar. 27 (1992), 241-242. MR 94h:52032

[18] M. Henk, Finite and Infinite Packings, Habilitationsschrift, Universität Siegen, 1995.

[19] M. Henk, Notes on covering minima and free planes, preprint.

[20] M. Henk and C. Zong, Segments in ball packings, Mathematika, in press.

[21] M. Henk, G. M. Ziegler and C. Zong, On free planes in lattice ball packings, Bull. London Math. Soc., in press.

[22] A. Heppes, Ein Satz über gitterförmige Kugelpackungen, Ann. Univ. Sci. Budapest Sect. Math. 3-4 (1960/61), 89-90. MR 24:A3562

[23] A. Heppes, On the number of spheres which can hide a given sphere, Canad. J. Math. 19 (1967), 413-418. MR 35:875

[24] I. Hortobágyi, Durchleuchtung gitterformiger Kugelpackungen mit Lichtbündeln, Studia Sci. Math. Hungar. 6 (1971), 147-150. MR 50:1131

[25] J. Horváth, Über die Durchsichtigkeit gitterförmiger Kugelpackungen, Studia Sci. Math. Hungar. 5 (1970), 421-426. MR 45:7615

[26] J. Horváth, On close lattice packing of unit spheres in the space $E^{n}$, Proc. Steklov Math. Inst. 152 (1982), 237-254.

[27] J. Horváth, Several Problems of $n$-dimensional Discrete Geometry, Ph.D. Thesis, Steklov Math. Inst., Moskow, 1986.

[28] J. Horváth, Eine Bemerkung zur Durchleuchtung von gitterförmigen Kugelpackungen, Proc. Internat. Conf. Geom., Thessalohiki, 1996, 187-191. MR 98h:52034

[29] G. A. Kabatjanski and V. I. Levenstein, Bounds for packings on a sphere and in space, Problems Inform. Transmission 14 (1978), 1-17.

[30] R. Kannan and L. Lovász, Covering minima and lattice-point-free convex bodies, Ann. Math. 128 (1988), 577-602. MR 89i:52020

[31] A. N. Korkin and E. I. Zolotarev, Sur les formes quadratiques positives quaternaires, Math. Ann. 5 (1872), 581-583.

[32] A. N. Korkin and E. I. Zolotarev, Sur les formes quadratiques positives, Math. Ann. 11 (1877), 242-292.

[33] K. Mahler, On the minimum determinant and the circumscribed hexagons of a convex domain, Proc. Kon. Ned. Akad. Wet. 50 (1947), 692-703. MR 9:10h

[34] J. Milnor and D. Husemoller, Symmetric Bilinear Forms, Springer-Verlag, Berlin, 1973. MR 58:22129

[35] S. P. Norton, A bound for the covering radius of the Leech lattice, Proc. Royal Soc. London A 380 (1982), 259-260. MR 84m:10022a

[36] R. A. Rankin, On positive definite quadratic forms, J. London Math. Soc. 28 (1953), 309-314. MR 14:1065g

[37] K. Reinhardt, Über die dichteste gitterförmige Lagerung kongruenter Bereiche in der Ebene und eine besondere Art konvexer Kurven, Abh. Math. Sem. Hamburg 10 (1934), 216-230.

[38] C. A. Rogers, A note on coverings and packings, J. London Math. Soc. 25 (1950), 327-331. MR 13:323b

[39] C. A. Rogers, The packing of equal spheres, Proc. London Math. Soc. 8 (1958), 609-620. MR 21:847

[40] C. A. Rogers, Lattice coverings of space, Mathematika 6 (1959), 33-39. MR 23:A2130

[41] C. A. Rogers, Packing and Covering, Cambridge University Press, Cambridge, 1964. MR 30:2405

[42] S. S. Ryskov and E. P. Baranovskii, Solution of the problem of least dense lattice covering of five-dimensional space by equal spheres, Soviet Math. Dokl. 16 (1975), 586-590. MR 55:273

[43] S. S. Ryskov and J. Horváth, Estimation of the radius of a cylinder that can be embedded in every lattice packing of n-dimensional unit balls, Math. Notes 17 (1975), 72-75. MR 51:6600

[44] C. L. Siegel, A mean value theorem in the geometry of numbers, Ann. Math. (2) 46 (1945), 340-347. MR 6:257b 
[45] I. Talata, On translational clouds for a convex body, Geom. Dedicata 80 (2000), 319-329. MR 2001f:52041

[46] R. T. Worley, The Voronoi region of $E_{6}^{*}, J$. Australian Math. Soc. Ser. A 48 (1987), 268-278. MR 88i: 11040

[47] R. T. Worley, The Voronoi region of $E_{7}^{*}$, SIAM J. Algebraic \& Discrete Methods 1 (1988), 134-141. MR 89k:11055

[48] C. Zong, Strange Phenomena in Convex and Discrete Geometry, Springer-Verlag, New York, 1996. MR 97m:52001

[49] C. Zong, A problem of blocking light rays, Geom. Dedicata 67 (1997), 117-128. MR 98g:52014

[50] C. Zong, A note on Hornich's problem, Arch. Math. 72 (1999), 127-131. MR 99k:52026

[51] C. Zong, Sphere Packings, Springer-Verlag, New York, 1999. MR 2000g:52020

[52] C. Zong, Simultaneous packing and covering in the Euclidean plane, Monatsh. Math. 134 (2002), 247-255. CMP 2002:08

[53] C. Zong, Simultaneous packing and covering in three-dimensional Euclidean space, J. London Math. Soc., in press.

School of Mathematical Sciences, Peking University, Beijing 100871, P. R. China

E-mail address: cmzong@math.pku.edu.cn 